\title{
Integration of Entropy Maximization and Quantum Behaved Particle Swarm Algorithm for Unsupervised Change Detection of MR Skull Bone Lesions
}

\author{
Ankita Mitra \\ Department of Electronics and \\ Communication, National \\ Institute of Technology, \\ Durgapur, India
}

\author{
Arunava De \\ Department of Information \\ Technology, Dr.B.C.Roy \\ Engineering College, \\ Durgapur, India
}

\author{
Anup Kumar \\ Bhattacharjee \\ Department of Electronics and \\ Communication, National \\ Institute of Technology, \\ Durgapur, India
}

\begin{abstract}
Entropy is the measure of randomness in a system whereas the entropy maximization procedure leads to the most probable state of a system behaviour.Entropy maximization using an optimization algorithm is used to find the threshold of the MR image of the brain. Standard Particle Swarm algorithm sufferes from stagnation. An automatic regrouping mechanism is used to deal with the stagnation. An Quantum Particle Swarm algorithm together with Entropy maximization helps us to get the most probable threshold value which correctly segments the lesions from the background in MR of brain. Using change detection algorithm the segmented object of the MR at time $t_{x}$ is compared with another object of the MR at the time $t_{y}$. The proposed method is applied on variety of MR images having lesions and gives favourable results in identifying changes taking place in the human brain.
\end{abstract}

\section{General Terms}

Change detection, Magnetic Resonance Imaging, Hybrid Particle Swarm Algorithm

\section{Keywords}

Region of Interest, Particle Swarm Optimization, Magnetic Resonance Imaging, Entropy, Hybrid Particle Swarm Optimization, Wavelet Mutation, Image Differencing

\section{INTRODUCTION}

In the field of medical image processing correct diagnosis of a diseased condition is an integral part of the treatment methodology. Also identifying minute tissue growth or absence of the same may lead to bigger ramifications which may be useful to curb the spread of the disease. Pathology is the study and diagnosis of a diseased condition. The main branches of pathology are clinical pathology, anatomical pathology or a combination of the two which is also referred to as general pathology. In pathology the extent of the spread of the disease can be identified.

The MR images used here in the paper are in Axial T2 view which primarily gives the pathology of the disease. The imaging was done on the same 1.5-Tesla MR imaging device provided by GE Medical. The images were viewed using centricity di-com viewer provided by GE Medical. The slice thickness was $5.0 \mathrm{~mm}$ and the gap between two slices was 1.5 $\mathrm{mm}$. Each slices having a resolution of $256 \times 256$.

The MR images are multi-modal images.Histogram of multimodal images have multiple peaks and valleys. Thus Multimodal images has multiple objects and backgrounds and hence multiple thresholds. The lesions in the multi-modal MR image can be correctly segmented using a single threshold or a combination of thresholds. This paper proposes a scheme of unsupervised change detection which integrates the principles of Entropy maximization using Quantum Particle Swarm Optimization algorithm also referred as QPSO[1]. The proposed framework is based on levels of intensity, because lesion portion of the MR image would have a different value of intensity as compared with an MR image without lesions. After the process of segmentation the segmented image is subjected to difference in Gaussians to enhance the edges. After the enhancement change detection algorithm is applied.

Particle swarm algorithm [2] is a stochastic optimization algorithm. Ref [3] found that Entropy Maximization using PSO algorithm gives better results for MR image segmentation. PSO suffers from stagnation which is a premature convergence of the particles in the Swarm. To overcome the deficiencies of PSO algorithm a Hybrid Quantum based PSO proposed by Sun [1] is used.

\section{RELATED WORK}

The histogram of multi-modal images has multiple peaks as opposed to two peaks for a bi-modal image. A.De et.al [3] proposed an Entropy Maximization based MR segmentation method. Hybrid Particle Swarm Optimization with Wavelet Mutation was used for optimization purpose. Reference [4] devised a technique to segment a diseased MRI image wherein the diseased part is segregated using a masking based thresholding technique together with entropy maximization. The particle swarm optimization technique (PSO) is used to get the region of interest (ROI) of the MRI image. The mask used is a variable mask. Reference [4] found that using Entropy maximization using PSO algorithm gives better results in comparison with other methods [5]. The problem addressed in [5] is the automatic segmentation of 
stroke lesions on MR multi-sequences. An energy criteria using intensity based class uncertainty and region homogeneity was formulated by [6]. Minimization of energy was done to select the threshold. Maximizing the between class variance in histogram based thresholding was done by [7]. However this method tends to split the larger part when the sizes of object and background are un-equal[8].

Reference [9] took advantage of the knowledge about the range of background proportion to the ROI to confine the range of threshold selection and achieved reliable results in segmenting magnetic resonance (MR) and computed tomography (CT) images of the human brain. Reference [10] demonstrated that threshold can be obtained by optimizing the weighted sum of the within-class variance and the intensity contrast. Ref.[11] review the main approaches to automated multiple sclerosis (MS) lesion segmentation. The key algorithmic contributions of [12] are the definition of an NL(non-local) data term and an NL regularization term to efficiently handle intensity inhomogeneities and noise in the data. The resulting new energy formulation is then built into an NL-Fuzzy C-Means (FCM) brain tissue segmentation algorithm. Reference [13] presents a framework of a medical image analysis system for the brain tumor segmentation and the brain tumor following-up over time using multi-spectral MRI images. Reference [14] presents a modified FCM algorithm for bias (also called intensity in-homogeneities) estimation and segmentation of MRI. Reference [15] introduces a rough neural intelligent approach for rule generation and image classification. Hybridization of intelligent computing techniques has been applied to see their ability and accuracy to classify breast cancer images into two outcomes: malignant cancer or benign cancer.

Reference [16] developed a method to segment high angular resolution diffusion imaging (HARDI) data. An automated scheme for magnetic resonance imaging (MRI) brain segmentation is proposed by [17]. An adaptive mean-shift algorithm clusters the joint spatial-intensity feature space, thus extracting a representative set of high-density points within the feature space, otherwise known as modes. Tissue segmentation is obtained by a follow-up phase of intensitybased mode clustering into the three tissue categories. Ref[18] built a appropriate model by considering spatial and temporal modes. The results provide accurate shape of the moving objects. A spatio-contextual unsupervised change detection technique for remote sensing images was used by [19]. The difference image was developed by using change vector analysis over a geographical area at different times. Gibb's Markov random field was used to model the spatial regularity between neighbouring pixels. An unsupervised change detection using a context sensitive technique was developed by [20]. This technique is based on Fuzzy clustering approach.

Ref[21] developed a MR image segmentation method is using Distance and Watershed Transforms. Several fuzzy and non-fuzzy histogram thresholding techniques are compared in
[22]. A longitudinal change detection framework for detecting modifications in diffusion MRI was proposed by [23]. A longitudinal change detection framework for detecting relevant modifications in diffusion MRI which were applied to neuromyelitis optica and multiple sclerosis was proposed by [24]. Stacks of medical images collected in time-series experiments were used to develop a tool for the visualization and statistical measurements of temporal changes of disease patterns by [25].

\section{PROPOSED ALGORITHM}

Transform coding is a procedure in which a function is subjected to an invertible transform which converts the statistically dependent elements of the function to independent coefficients. Hence the data are compressed by redundancy reduction. However to achieve further compression the transformed data are also subjected to irrelevancy reduction. Hence irrelevant data are discarded. Redundancy reduction is reversible but irrelevancy reduction is irreversible.

In this article the aim is to discard irrelevant details and concentrate on the region of the human organ where a change has taken place. The relevant sections are the lesions in the human brain. The brain image of the same subject is taken at two separate times and is compared using a suitable change detection algorithm.

Separating the lesion from the healthy sections of the human brain requires selection of a suitable threshold. The image under consideration is a Magnetic Resonance Image and hence it has multiple objects and background. Multiple objects and background leads to the conclusion that MR image of brain has multiple thresholds. After selecting a suitable threshold that correctly segregates the lesion from the object, it has to be suitably optimized using a suitable optimization algorithm.

Segmentation using this method works better if we can identify the foreground objects and background locations. Our segmentation follows the below basic procedure:

\subsection{Entropy Maximization}

A normalized histogram of a gray scale image is computed. [26]

$$
P_{n}=h(n)=f_{n} / N, n=0,1,2, \ldots 255
$$

$f_{n}$ is the observed frequency of gray level $n$. For multimodal images the image is divided into $(\mathrm{k}+1)$ number of homogeneous zones having threshold gray levels at $\mathrm{t}_{1}, \mathrm{t}_{2}, \mathrm{t}_{3}, \ldots \mathrm{t}_{\mathrm{k}}$.

The function $\mathrm{H}$ which incidentally is the histogram function of the gray level image is maximized to get a threshold value. The threshold value may be in the range say Tx to Ty. The threshold value is further optimized using QPSO (Quantum behaved Particle Swarm Algorithm) which is explained in the subsequent sections. 


\subsubsection{Particle Swarm Optimization Algorithm and its Drawbacks:}

Particles are agents moving in a search space with DimensionD. The set of these particles are called a Swarm. The particle is described by the following parameters position, velocity and the best position found so far.

The particle Swarm algorithm is a stochastic algorithm. The PSO algorithm has a drawback that it suffers from the problem of premature convergence. In the early stages of algorithm execution the convergence of the particles is sharp whereas in the later stages of execution it saturates and may even terminate. If the position of the particle at a given time coincides with the global best position then the particle will move away from this point if particle's inertia weight and velocity are different from zero. The particle will stop moving if the velocities are zero and are close to global best particle and thus it leads to premature convergence and thus further improvement cannot be achieved. This phenomena is called "Stagnation" [26].

Though Quantum based Particle Swarm Algorithm (QPSO) [1] out-performs the standard PSO and also has fewer control parameters, it still suffers from the phenomena of stagnation. In Quantum based Particle Swarm Optimization Algorithm the state of the particles are determined by the quantum model. The parameters $w, c_{1}, c_{2}$ and the velocity term in the Particle Swarm Optimization algorithm are eliminated in QPSO [27].

\subsection{Quantum Behaved Particle Swarm Algorithm:}

Ref [28] states that each particle would converge to its local attractor $p_{\mathrm{i}}$ where $p_{i}$ is treated as a stochastic attractor of a particle $\mathrm{i}$ which lies in the hyper-rectangle with pbest $_{\mathrm{i}}$ and gbest.

$$
p_{i, j}=\emptyset \cdot \text { pbest }_{i, j}+(1-\emptyset) \cdot \text { gbest }_{j}
$$

Where $\emptyset \in(0,1)$

Based on the above characteristics Sun et al. [1] proposed a quantum behaved Particle Swarm Optimization (QPSO) algorithm. In this algorithm each particle is devoid of the velocity vector and has only the position vector.

The position is updated using the following formula[27]:

$$
\begin{aligned}
& \operatorname{Pos}_{i, j}(t+1)= \\
& \left\{\begin{array}{l}
p_{i, j}(t)+\beta \cdot\left(\operatorname{Sbest}_{j}(t)-\operatorname{Pos}_{i, j}(t)\right) \cdot \ln \left(\frac{1}{v}\right), \text { if } h>0.5 \\
p_{i, j}(t)-\beta \cdot\left(\operatorname{Sbest}_{j}(t)-\operatorname{Pos}_{i, j}(t)\right) \cdot \ln \left(\frac{1}{v}\right), \text { otherwise }
\end{array}\right.
\end{aligned}
$$

$v$ is a random number in the range $(0,1)$ which are distributed uniformly. $\beta$ is used to control the speed of convergence of the algorithm and is called contraction expansion coefficient. Sbest is called the mean of best position of the population and is calculated using the following equation:

$$
\operatorname{Sbest}_{j}(t)=\frac{1}{N} \sum_{i=1}^{N} \text { pbest }_{i j}(t)
$$

The QPSO algorithm is defined by the following steps[27]: 1.Begin

2. Start of loop condition Fe's $<=$ MaxFe's

3. For each particle $i$ do

4. Update the position according to (3);

5. Fitness value of the new particle is calculated;

6. Fe's++

7. end for.

8.Update the $p$ best, $g$ best and $p$ in the population.

9. End of outer loop

10. End.

Where $p$ is the local attractor, Fe's are the number of fitness evaluations and MaxFe's is the maximum number of Fe's. QPSO does not have a velocity term as also the parameters $c_{1}$ and $\mathrm{c}_{2}$.

QPSO introduced a new operator $\beta$ which is reported in some literatures that it linearly decreases from 1.0 to $0.5[1,29]$. We use Entropy Maximization together with QPSO to get the threshold value for segmenting the lesions of MR image of brain. Using the above discussed method and expert knowledge the value of the threshold was found to be 160 for segmenting lesions for a gray scale MR image of brain. Fig.2(a) and(b) illustrates that the lesions present in Fig.1(a) and (b) are correctly segmented using this method.

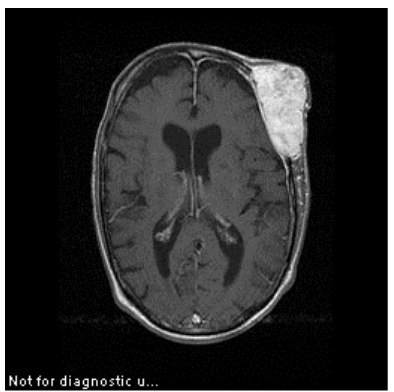

a)

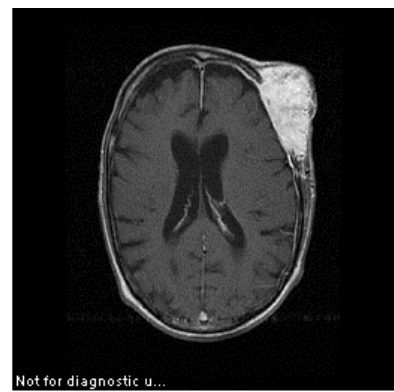

b)
Figure 1 a) Sample Image-1 from Dataset Fig.5 b) Sample Image-2 from Dataset Fig.5

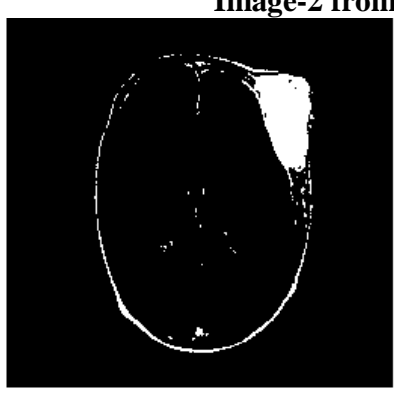

a)

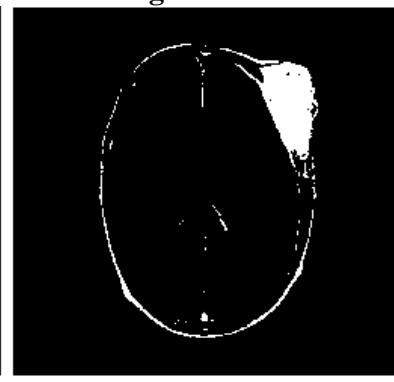

b)
Figure 2 Segmentation of figure1 (a) and figure1(b) using QPSO

$N$ is defined as the size of the population. 


\subsection{Difference of Gaussians}

Difference of Gaussians is a feature enhancement filter used for edge enhancement. This filter involves the subtraction of one smoothed version of the image with another less smoothed version of the image. The smoothed images are obtained by convolving the original image with Gaussian filters with kernel weights. Two different standard deviations (i.e. Gaussian Distributions) determine the kernel weights. High frequency spatial information is suppressed when an image is smoothed by Gaussian Kernel. Spatial information lying between a range of frequencies are preserved in the two images when one image is subtracted from another. Thus it behaves as a band-pass filter that only passes frequencies within a certain range [30].

The algorithm operates by differencing the results of convolving two kernels of weights with each grid cell and its neighbours in an image. The 2 Dimensional Gaussian Curve determines the weights of the convolution kernels. Gaussian curve gives stronger weighting to cells nearer the kernel centre. Two standard deviation parameters determine the size of the convolution kernels. The second standard deviation should be larger than the first. Both standard deviations can range from 0.5 to 20 [30].

Edge enhancement algorithms often increase the random noise in the image which is unwanted. Difference of Gaussian involves removal of High Frequency Spatial detail including random noise.

Given a m-channels, n-dimensional image [31]

$$
I:\left\{X \subseteq R^{n}\right\} \rightarrow\left\{Y \subseteq R^{m}\right\}(5)
$$

The difference of Gaussians of the image I is the function

$$
\Gamma_{\sigma 1 \sigma 2}:\left\{X \subseteq R^{n}\right\} \rightarrow\{Z \subseteq R\}(6)
$$

Obtained by subtracting the image I convolved with the Gaussian of variance $\sigma_{2}^{2}$ from the image I convolved with a Gaussian of narrower variance $\sigma_{1}^{2}$, with $\sigma_{2}>\sigma_{1}$. In one dimension, $\Gamma$ is defined as:

$$
\Gamma_{\sigma 1 \sigma 2}(x)=I * \frac{1}{\sigma_{1} \sqrt{2 \pi}} e^{\frac{-\left(x^{2}\right)}{\left(2 \sigma_{1}^{2}\right)}}-I * \frac{1}{\sigma_{2} \sqrt{2 \pi}} e^{\frac{-\left(x^{2}\right)}{\left(2 \sigma_{2}^{2}\right)}}(7)
$$

and for the centered two dimensional case:

$$
\Gamma_{\sigma, K \sigma}(x, y)=I * \frac{1}{2 \pi \sigma^{2}} e^{-\left(x^{2}+y^{2}\right) /\left(2 \sigma^{2}\right)}-I * \frac{1}{2 \pi K^{2} \sigma^{2}} e^{\frac{-\left(x^{2}+y^{2}\right)}{\left(2 K^{2} \sigma^{2}\right)}}
$$

Which is equivalent to :

$\Gamma_{\sigma, K \sigma}(x, y)=I *\left(\frac{1}{2 \pi \sigma^{2}} e^{-\left(x^{2}+y^{2}\right) /\left(2 \sigma^{2}\right)}-\frac{1}{2 \pi K^{2} \sigma^{2}} e^{\frac{-\left(x^{2}+y^{2}\right)}{\left(2 K^{2} \sigma^{2}\right)}}\right.$

Which represents an image approximating a Mexican Hat function. Difference of Gaussians is used to enhance the edges and to highlight other details of the image[31]. Difference of Gaussians is used to enhance the edges of the image. The Fig.3(a) depicts the edge enhancement of the segmented image of Fig.2(a) and Fig.3(b) depicts its inverse.

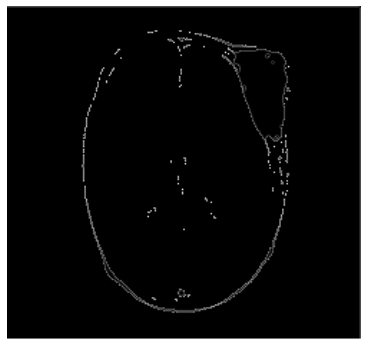

a)

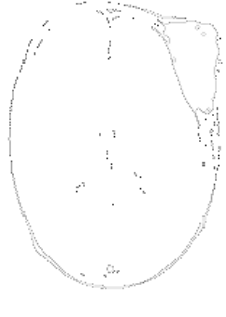

b)
Figure 3 a) Enhanced edges of figure2(a) using Difference of gaussians b) Inverse of figure.3(a)

\subsection{Change Detection}

Change detection methods are based on signed difference images. The thresholding is done on the difference image[32].The change mask is generated according to the following equation[33]:

$$
E(x)=\left\{\begin{array}{c}
1, \text { if }|E(x)|>\tau \\
0, \text { otherwise }
\end{array}\right\}
$$

$\tau$ is the threshold which is chosen empirically. This algorithm is the algorithm viewed by us to detect the progress of lesions in MRI of brain. The change detection difference map is depicted in Fig.4(a) and its inverse Fig.4(b).

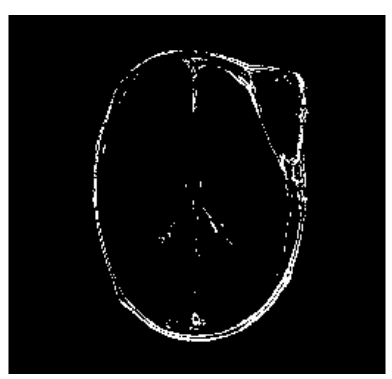

a)

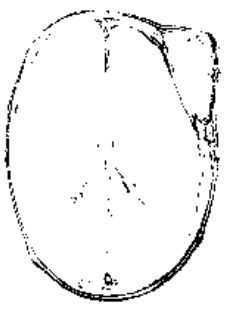

b)
Fig.4 a)Difference Map b) Inverse of Difference Map

\section{DISCUSSIONS AND CONCLUSIONS}

We have done quantitative analysis of sample data Fig.1(a) and Fig.1(b) from the dataset Fig.5. Fig.5 classifies the 19 slices as relevant and non-relevant. Relevant cases are those which has lesions in them. The MR images used are in Axial $\mathrm{T} 2$ view gives information on the pathology of the disease.

A study of change detection results using QPSO and PSO is made. PSO and QPSO both suffered from stagnation but QPSO out-performs the standard PSO and also has fewer control parameters. In QPSO algorithm the state of the particles are determined by the quantum model. The parameters $w, c_{l}, c_{2}$ and the velocity term in the PSO algorithm are eliminated in QPSO.

Analysis is carried out in terms of overall error (OE), missed alarms (MA), false alarms (FA). False alarm is defined as unchanged pixels categorized as changed ones. Missed alarm is defined as changed pixels labelled as unchanged ones. For change detection applications it is desirable to have less MA since it is the actual changes that the algorithm failed to 
detect. Overall error should be minimal. A comparison of $\mathrm{OE}$, MA, FA is made between the change detection algorithm based on QPSO and PSO. Table 1 enlists the results. It is thus clear that Change detection based on Entropy maximization using Quantum based PSO and Difference of Gaussians gives us better results compared with that based on Particle Swarm algorithm.

The algorithm used here for change detection can be used in variety of MR images of human body. Gall bladder and Tongue cancer can be detected using the change detection techniques discussed above.
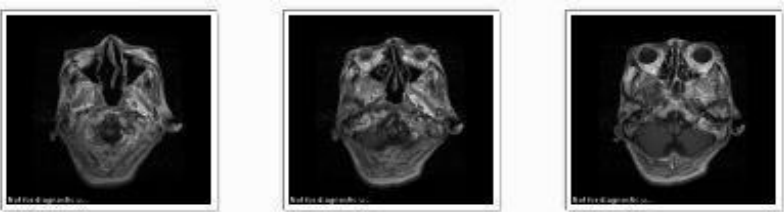

Slice-1 Label-0

Slice-2 Label-0

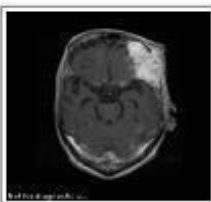

Slice-7 Label-1

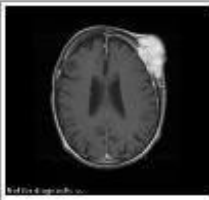

Slice-13 Label-1

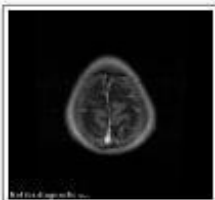

Slice-19 Label-0

Label-0 Not Relevant Case

Label-1 Relevant Case

TABLE 1 Change detection Results for the sample dataset of Figure1

\begin{tabular}{|c|c|c|c|}
\hline Techniques Used & MA & FA & OE \\
\hline $\begin{array}{c}\text { Using Particle } \\
\text { Swarm Algorithm }\end{array}$ & 2301 & 1727 & 4028 \\
\hline $\begin{array}{c}\text { Using Quantum } \\
\text { Based Particle } \\
\text { Swarm Algorithm }\end{array}$ & 2120 & 932 & 3052 \\
\hline
\end{tabular}

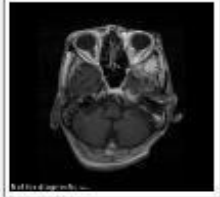

Slice-4 Label-0

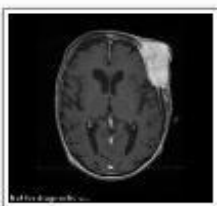

Slice-10 Label-1

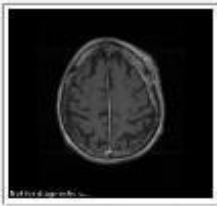

Slice-16 Label-1
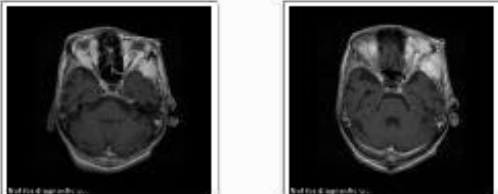

Slice-5 Label-0

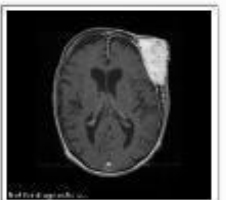

Slice-11 Label-1

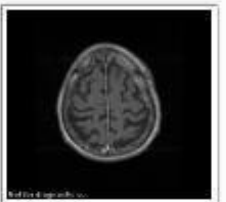

Slice-17 Label-0
Slice-6 Label-1

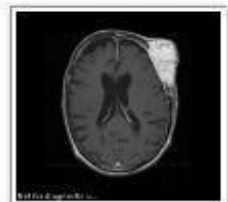

Slice-12 Label-1

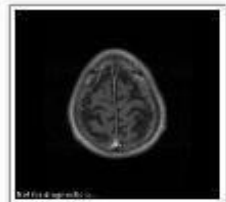

Slice-18 Label-0

Figure 5 Patient Dataset

\section{REFERENCES}

[1] J. Sun, B. Feng, and W. B. Xu, "Particle swarm optimization with particles having quantum behavior," in Proceedings of the Congress on Evolutionary Computation (CEC '04), pp. 325-331, June 2004.

[2] Kennedy,J., Eberhart,R.,"Particle Swarm Optimization",Proc. of IEEE International Conference on Neural Networks, Vol.4,pp.1942-1948,1995

[3] Arunava De, Anup Kumar Bhattacharjee, Chandan Kumar Chanda, Bansibadan Maji, "MRI Segmentation using Entropy Maximization and Hybrid Particle Swarm
Optimization with Wavelet Mutation”, Proceedings of World Congress on Information and Communication Technologies (WICT 2011), Mumbai, pp. 362-367, December 2011.

[4] Arunava De, Rajib Lochan Das, Anup Kumar Bhattacharjee, Deepak Sharma," Masking based segmentation of diseased MRI images", Proc. of International Conference on Information Science and Applications, IEEE Seoul chapter, Seoul,Korea, pp.230236, Aug. 2010 
[5] Y.Kabir, M.Dojat, B.Scherrer, F.Forbes,C.Garbay," Multimodal MRI Segmentation of Ischemic Stroke lesions" , Proc.of the $29^{\text {th }}$ Annual International Conference of the IEEE EMBS, Cite Internationale, Lyon France, August 23- 26, 2007.

[6] P.K. Saha and J.K. Udupa, "Optimum image thresholding via class uncertainty and region homogeneity", IEEE Trans. Pattern Anal. Mach. Intell., vol .23, no .7,pp . 689-706, 2001.

[7] N. Otsu, "A thresholding selection method from graylevel histograms”, IEEE Trans. Syst. Man Cybern, vol .9, no . 1,pp.62-66,1979.

[8] J. Kittler and J. Illingworth, "On threshold selection using clustering criteria", IEEE Trans. Syst. Man Cybern., vol. 15, no. 5, pp.652-655,1985.

[9] Q. Hu,Z. Hou and W.L. Nowinski, "Supervised rangeconstrained thresholding", IEEE Trans. Image Process, vol . 15,no . 1,pp . 228-240,2006.

[10] Y. Qiao, Q. Hu, G. Qian, S. Luo, and W. L. Nowinski,"Thresholding based on variance and intensity contrast," Pattern Recognition, vol. 40, pp. 596 - 608, 2007.

[11] Xavier Llado,Arnau Oliver, Mariano Cabezas, Jordi Freixenet, Joan C.Vilanova, Ana Quiles, Laia Valls, Lluis Ramio-Torrenta, Alex Rovira,"Segmentation of multiple sclerosis lesions in brain MRI: A review of automated approaches",Information Sciences, Vol. 186,no.1,pp.164-185,2012.

[12] Benoit Caldairou, Nicolas Passat, Piotr A. Habas, Colin Studholme, Francois Rousseau, "A non-local fuzzy segmentation method: Application to brain MRI", Pattern Recognition, Vol. 44, no.9,pp.1916-1927,2011

[13] Nan Zhang, Su Ruan, Stephane Lebonvallet, Qingmin Liao, Yuemin Zhu, " Kernel feature selection to fuse multi- spectral MRI images for brain tumor segmentation", Computer Vision and Image Understanding, Vol.115,no.2,pp.256- 269, 2011.

[14] M.Y. Siyal, Lin Yu, ” An intelligent modified fuzzy cmeans based algorithm for bias estimation and segmentation of brain MRI", Pattern Recognition Letters, Vol.26, no.13,pp.2052-2062, 2005.

[15] Aboul ella Hassanien, Dominik Slezak,“ Rough neural intelligent approach for image classification: A case of patients with suspected breast cancer." International Journal of Hybrid Intelligent Systems, Vol. 3, no.4, pp. 205-218, 2006.

[16] Maxime_Descoteaux, Rachid_Deriche, "High Angular Resolution Diffusion MRI Segmentation Using RegionBased Statistical Surface Evolution”, Journal of Mathematical Imaging and Vision,Vol.33(2),pp 239252,2009
[17] Arnaldo Mayer and Hayit Greenspan, "An Adaptive Mean-Shift Framework for MRI Brain Segmentation”, IEEE Transactions on Medical Imaging, Vol. 28 (8), 2009.

[18] B.N. Subudhi , S. Ghosh ,A.Ghosh, "Change detection for moving object segmentation with robust background construction under Wronskian framework", Machine Vision and Applications,pp.795 - 809,2013

[19] A.Ghosh, B.N. Subudhi and L. Bruzzone, Integration of Gibbs Markov Random Field and Hopfield-Type Neural Networks for Unsupervised Change Detection in Remotely Sensed Multi-temporal Images, IEEE Transactions on Image Processing, Vol. 22, No. 8, 2013.

[20] Ashish Ghosh, Niladri Shekhar Mishra, Susmita Ghosh, "Fuzzy clustering algorithms for unsupervised change detection in remote sensing images", Information Sciences, Vol.181(4),pp-699-715, 2011.

[21] A.Mitra et al," MRI Skull Bone Lesion segmentation using Distance based Watershed Segmentation", Proc. of the International Conference on Frontiers of Intelligent Computing: Theory and Applications (FICTA), Springer Advances in Intelligent Systems and Computing, Vol. 328, pp 255-261, 2015

[22] Swarnajyoti Patra, Susmita Ghosh \& Ashish Ghosh, "Histogram thresholding for unsupervised change detection of remote sensing images", International Journal of Remote Sensing,Vol.32(21),2011

[23] Grigis A. et al., "Change detection in diffusion MRI using multivariate statistical testing on tensors", International Conference on Medical Image Computing and Computer Assisted Intervention, 2010

[24] Grigis A. et al., "Longitudinal change detection in diffusion MRI using multivariate statistical testing on tensors", NeuroImage,vol.60(4),pp-2206-21,2012

[25] Jung Soh , Mei Xiao , Thao Do , Oscar Meruvia-Pastor , Christoph W. Sensen ,"Integrative Visualization of Temporally Varying Medical Image Patterns",Journal of Integrative Bioinformatics, Vol.8(2),2011.

[26] A.De et al.,"Hybrid Particle Swarm Optimization with Wavelet Mutation based Segmentation and Progressive Transmission Technique for MRI Images", International Journal of Innovative Computing, Information and Control, Japan, Vol.8,no.7(B),pp. 5179-5197, July 2012.

[27] Xioa Fu, Wangsheng Liu, Bin Zhang, Hua Deng, "Quantum Behaved Particle Swarm Optimization with Neighborhood Search for Numerical Optimization", Mathematical Problems in Engineering, Vol.2013, Article ID 469723, pp.1-10 
[28] F. van den Bergh and A. P. Engelbrecht, "A study of particle swarm optimization particle trajectories," Information Sciences, vol. 176, no. 8, pp. 937-971, 2006.

[29] J. Sun, W. B. Xu, and W. Fang, “A diversity-guided quantum behaved particle swarm optimization algorithm," in Simulated Evolution and Learning, vol. 4247 of Lecture Notes in Computer Science, pp. 497-504, Springer, New York, NY,USA, 2006.

[30] Tutorials on Difference of Gaussians, www.uoguelph.ca/ hydrogeo/Whitebox/Help/FilterDoG. html
[31] Tutorials on Difference of Gaussians, pediaview.comOpen Source Encyclopedia.

[32] A.Mitra et al," Detection of Progression of Lesions in MRI Using Change Detection”, Proc. of the International Conference on Frontiers of Intelligent Computing: Theory and Applications (FICTA), Springer Advances in Intelligent Systems and Computing Vol.247, pp.467-473, Nov. 2013.

[33] Radke, R.J., Andra, S., Al-Kofahi, O., Roysam, B.: Image Change Detection Algorithms: A Systematic Survey. IEEE Trans. Image. Process 14, 294-307 (2005). 